
\title{
25 Research Square \\ The added value of parenchymal transcranial Doppler sonography at the Emergency Room: case report of an unusual gait disorder
}

Massimiliano Godani

Sant'Andrea Civic Hospital, La Spezia

Giuseppe Lanza ( $\nabla$ giuseppe.lanza1@unict.it )

University of Catania https://orcid.org/0000-0002-5659-662X

Rita Bella

University of Catania

Lucia Trevisan

University of Genoa

Raffaele Ferri

Oasi Research Institute - IRCCS, Troina

\section{Case Report}

Keywords: Movement disorders, Huntington's disease, transcranial sonography, diagnosis, Emergency Medicine

Posted Date: December 4th, 2019

DOI: https://doi.org/10.21203/rs.2.17907/v1

License: (c) (i) This work is licensed under a Creative Commons Attribution 4.0 International License. Read Full License 


\section{Abstract}

Background: the growing application of parenchymal transcranial Doppler sonography (pTCS) helps the diagnosis of a wide range of neurological diseases, especially movement disorders. Here, we report a patient with an unusual gait disorder in whom pTCS performed at the Emergency Room rapidly provided diagnostic clues towards a degenerative movement disorder.

Case presentation: A 60-year old man presented at the Emergency Room with a rapidly progressive history of walking difficulty, without falls. He had no family history of neurodegenerative or psychiatric diseases. Because of a depressive disorder arisen one year earlier, he was under oral treatment with escitalopram $10 \mathrm{mg}$ and amisulpride $100 \mathrm{mg}$ daily. Neurological examination showed a gait characterized by ataxia, rigidity, and limping; he also had mild oro-facial and hands dyskinesia, some of which were also present during walking. Brain computed tomography was normal. A pTCS performed at the Emergency Room showed hyperechogenicity of the substantia nigra and the caudate nucleus, bilaterally. This pattern is suggestive of Huntington's disease (HD), as confirmed by the molecular genetic test carried out later. Other laboratory tests, standard electroencephalogram, and 1.5-T brain MRI were normal.

Conclusions: to date, this is the first report of HD sonographically detected at the Emergency Room. This supports the view that pTCS can be easily implemented in the diagnostic algorithm and differential diagnosis of movement disorders even in an urgency setting. Notably, conventional MRI did not detect, at this stage, any abnormality; this further highlights the diagnostic utility of pTCS, which also allowed to exclude a drug-induced effect or a different movement disorder. In clinically suspected cases, pTCS can extend the neurological examination by providing diagnostic clues more rapidly and less expensively than neuroimaging.

\section{Background}

Parenchymal transcranial Doppler sonography (pTCS) has become a reliable and sensitive diagnostic technique, enabling a two-dimensional visualization of the main brain structures through the temporal bone [1]. The growing application of this technique helps the diagnosis and differential diagnosis of a wide range of neurological diseases, especially movement disorders. pTCS is easily available and applicable, non-invasive, painless, cost effective, and quick to perform at the bedside, even in littlecollaborating patients or in those with involuntary movements because head motion can be well compensated by the operator, at least to some extent [2]. These features make pTCS a useful additional tool for movement disorders, particularly in the case of challenging diagnoses or doubtful clinical presentations. Moreover, subtle changes of specific deep brain structures, such as abnormal trace metal accumulation and circumscribed degenerative processes, can often be detected more sensitively with pTCS than with magnetic resonance imaging (MRI) [3,4]. The recently developed TCS machines [5] have offered the possibility to display some structures (e.g. basal ganglia, mesencephalic nuclei, width of the 
third ventricle and the frontal horns of lateral ventricles) with a very high lateral and axial resolution, similar to that of MRI in clinical applications [6].

However, despite the above mentioned advantages, to date pTCS is still considered as an elective exam to perform in selected in-patients only, especially in those with movement disorders. Actually, although movement disorders are commonly viewed as chronic diseases that are followed and treated in ambulatory care setting, a growing number of these patients present with acute or subacute disabling symptoms, as well as with complications of their underlying condition. Moreover, an acute movement disorder presenting at the Emergency Room (ER) points to a number of systemic, neurologic, druginduced, or sometimes functional processes. In this context, a delayed diagnosis often leads to a significant distress for the patients, with consequences on their management and quality of life $[7,8]$. Here, we report a patient with a rapidly progressive gait disorder in whom pTCS performed at the ER provided diagnostic findings towards a neurodegenerative movement disorder.

\section{Case Presentation}

A 60-year-old man presented at the ER because of a two-week history of progressive walking difficulty, without falls. Family history was negative for neurological or psychiatric diseases. He worked as an electrician and lived alone. Because of a depressive disorder arisen one year earlier, he was under stable treatment with oral escitalopram $10 \mathrm{mg}$ and amisulpride $100 \mathrm{mg}$ daily. He was not taking any other medication, including the weeks before his presentation at the hospital.

Neurological examination showed an unusual gait characterized by ataxia, rigidity, and limping (see video), with some instability but without falls. He also had mild oro-facial and hands dyskinesia, some of which were also present during walking, whereas the rest of the clinical exam was normal. Urgent laboratory tests, electrocardiogram, and computed tomography (CT) scan were normal. Based on the clinical picture, it was decided to perform a pTCS at the ER. The exam showed hyperechogenicity of the substantia nigra (SN) and the caudate nucleus, bilaterally (fig. 1). This pTCS pattern was suggestive of a degenerative movement disorder, namely Huntington's disease (HD). Despite the lack of a family history and the late onset of symptoms, HD was considered as the most probable cause accounting for his clinical manifestations. Therefore, he was admitted to the Neurology Unit and underwent electroencephalogram, 1.5-T brain MRI, and extensive blood tests, which did not reveal any pathological finding. Molecular genetic test confirmed the diagnosis of HD, with one allele of the huntingtin (HTT) gene in chromosome $4 \mathrm{p} 16.3$ showing an abnormal number of $40 \pm 1$ cytosine-adenine-guanine (CAG) repeats, whereas the number of CAG repeats in the other allele was within the normal limit $(24 \pm 1)$.

\section{Discussion And Conclusion}

To date, this is the first report of a HD sonographically detected at the ER. This supports the view that pTCS can be easily implemented in the diagnostic algorithms and differential diagnosis of movement disorders, even in urgency settings [9]. 
$\mathrm{HD}$ is an autosomal dominant disorder caused by an unstable expansion of the trinucleotide CAG on chromosome 4p16.3, leading to a progressive degeneration of the basal ganglia, particularly of the neostriatum [10]. The diagnosis focuses on typical involuntary movements, although the clinical presentation also includes cognitive decline and psychiatric symptoms (i.e. depression, irritability, anxiety, psychosis, and compulsive behavior) [11]. Psychiatric disturbances often precede the onset of motor symptoms [12], as in the present case, thus challenging an early diagnosis and treatment. Neuroimaging methods, such as CT and MRI, may detect structural abnormalities, although they often report normal findings, especially in the early stages [13]. Indeed, the MRI of our patient did not detect any abnormality, thus highlighting the diagnostic utility of pTCS that might be more sensitive of MRI in early HD. Nuclear imaging methods, i.e. single photon emission tomography and positron emission tomography, can identify biochemical changes in the basal ganglia and brainstem [14], but they are expensive and not widely accessible.

Although the frequency of late-onset HD ( $>59$ years) is assumed to be low and the clinical course milder, the previous literature is scarce and often inconclusive. A very recent study on a large cohort [15] founds that late-onset patients present more frequently with gait and balance problems as first symptoms, and the disease progression is not milder compared to common-onset HD patients, apart from motor progression. Moreover, in this patient subgroup, the family history is likely to be negative, which might make diagnosing HD more difficult, although balance and gait problems are helpful clinical clues [15]. Taken together, these findings are in line with the patient described here; in this context, it is likely that gait and motor disorder had been present for more than two weeks, although neither the patient nor the family members reported them.

The pTCS finding of SN hyperechogenicity indicates a functional impairment of the nigro-striatal dopaminergic system [4], and is assumed to be based on an increased amount of iron bound to proteins other than ferritin $[4,16]$. Although SN hyperechogenicity is characteristic for idiopathic Parkinson's disease (PD), it has been reported also in other conditions, such as atypical parkinsonism, spinocerebellar ataxia, and HD [17-19]. In particular, Postert and colleagues [17] observed SN hyperechogenicity in 12 out of 45 patients with HD (27\%), of the caudate nuclei in 6 (13\%), and of the lentiform nuclei in 4 (9\%). This condition leads to changes of iron-protein binding, which may cause echogenic alterations similar to the SN hyperechogenicity observed in PD [4]. This is also in agreement with molecular studies indicating the essential role of huntingtin in cellular iron homeostasis [20].

We also can confirm the pathologic signal of the caudate nuclei in $\mathrm{HD}$, a finding that supports the discrimination from other movement disorders [21]. An association between caudate nuclei hyperechogenicity and increased signal intensity in T2-weighted MRI in HD has been reported [17]. However, the precise morphological interpretation is still speculative and further neuropathological and multimodal imaging studies are needed. Recently, Rosas and coworkers [22] performed functional MRI and mass spectrometry to demonstrate increased iron levels in the basal ganglia and cortical structures both in presymptomatic gene carriers and HD patients. 
Finally, it is worth mentioning that this patient was taking amisulpride, a benzamide blocking or antagonizing the presynaptic dopamine D2 receptor. As such, amisulpride is among the neuroactive compounds able to induce tardive dyskinesia, although the prevalence of amisulpride-induced movement disorder is substantially lower than that of the first-generation antipsychotic agents. Moreover, tardive dyskinesias commonly manifests with oro-facial movements, tremor, dystonia, akathisia, and parkinsonism [23]. Since oro-facial dyskinesia was present also in the present case, a iatrogenic cause needed to be taken into account. However, the execution of PTCS at the ER allowed to detect clear signs of an underlying neurodegenerative process and, concomitantly, to exclude a drug-induced effect (where pTCS is normal).

As known, pTCS has several limitations, including the temporal acoustic bone window (insufficient in 5$10 \%$ of caucasian individuals [4]), the operator expertise, and the quality of the ultrasound system. Therefore, reference values need to be obtained specifically for each TCS system and laboratory [2].

In conclusion, in clinically suspected cases, pTCS can feasibly extend the neurological examination by providing diagnostic clues more rapidly and less expensively than neuroimaging. Further systematic studies are needed to confirm the clinical diagnostic utility of pTCS in patients with movement disorders presenting at the ER.

\section{List Of Abbreviations}

CAG: cytosine-adenine-guanine

CT: computed tomography

ER: Emergency Room

HD: Huntington's disease

MRI: magnetic resonance imaging

PD: Parkinson's disease

SN: substantia nigra

pTCS: parenchymal transcranial Doppler sonography

\section{Declarations Section}

Ethics approval and consent to participate: the patient gave his written informed consent prior to the inclusion in the study, in accordance with the ethical standards laid down in the 1964 Declaration of Helsinki and its later amendments 
Consent to publish: the patient gave his written informed consent for the publication of his personal and medical information.

Availability of data and materials: all data generated or analyzed during this study are included in this published article

Competing interests: the authors declare no competing interests

\section{Funding: none}

Authors' Contributions: all authors: a) made substantial contributions to conception and design, as well as to acquisition and interpretation of data; $b$ ) were involved in drafting the manuscript and revising it critically; c) participated sufficiently in the work to take public responsibility for appropriate portions of the content and given final approval; $d$ ) ensured that questions related to the accuracy or integrity of any part of the work have been appropriately investigated. In particular: MG recruited the patient and dealt with the acquisition and interpretation of data; GL designed the study and write the first draft of the manuscript; RB reviewed the literature and critically revised the draft; RF drafted and finalized the manuscript.

Acknowledgements: we would like to thank the Italian Society of Neurosonology and Cerebral Hemodynamic (SINSEC) for education and training.

\section{References}

1. Seidel G, Kaps M, Gerriets T, Hutzelmann A. Evaluation of the ventricular system in adults by transcranial duplex sonography. J Neuroimaging. 1995;5:105-8.

2. Berg D, Godau J, Walter U. Transcranial sonography in movement disorders. Lancet Neurol. 2008;7:1044-55. doi: 10.1016/S1474-4422(08)70239-4.

3. Becker G, Berg D. Neuroimaging in basal ganglia disorders: perspectives for transcranial ultrasound. Mov Dis. 2001;16:23-32.

4. Walter U, Behnke S, Eyding J, Niehaus L, Postert T, Seidel G, et al. Transcranial brain parenchyma sonography in movement disorders: State of the art. Ultrasound Med Biol. 2007;33:15-25.

5. Go CL, Frenzel A, Rosales RL, Lee LV, Benecke R, Dressler D, et al. Assessment of SN echogenicity in German and Filipino populations using a portable ultrasound system. J Ultrasound Med. 2012;31: 191-6.

6. Walter U, Kanowski M, Kaufmann J, Grossmann A, Benecke R, Niehaus L. Contemporary ultrasound systems allow high-resolution transcranial imaging of small echogenic deep intracranial structures similarly as MRI: a phantom study. Neuroimage. 2008;40:551-8. doi:

10.1016/j.neuroimage.2007.12.019.

7. Dallocchio C, Matinella A, Arbasino C, Arno' N, Glorioso M, Sciarretta M, et al. Movement disorders in emergency settings: a prospective study. Neurol Sci. 2019;40:133-8. doi: 10.1007/s10072-018- 
3601-1.

8. Lanza G, Papotto M, Pennisi G, Bella R, Ferri R. Epileptic seizure as a precipitating factor of vascular progressive supranuclear palsy: a case report. J Stroke Cerebrovasc Dis. 2014;23:e379-81. doi: 10.1016/j.jstrokecerebrovasdis.2013.12.043.

9. Mijajlovic MD, Tsivgoulis G, Sternic N. Transcranial Brain Parenchymal Sonography in Neurodegenerative and Psychiatric Diseases. J Ultrasound Med. 2014; 33:2061-8. doi: 10.7863/ultra.33.12.2061.

10. Aylward EH, Sparks BF, Field KM, Yallapragada V, Shpritz BD, Rosenblatt A, et al. Onset and rate of striatal atrophy in preclinical Huntington disease. Neurology. 2004;63:66-72.

11. Paulsen JS, Ready RE, Hamilton JM, Hamilton JM, Mega MS, Cummings JL. Neuropsychiatric aspectsof Huntington's disease. J Neurol Neurosurg Psychiatry. 2001;71:310-4.

12. Duff K, Paulsen JS, Beglinger LJ, Langbehn DR, Stout JC; Predict-HD Investigators of the Huntington Study Group. Psychiatric symptoms in Huntington's disease before diagnosis: the predict-HD Study. Biol Psychiatry. 2007;62:1341-6.

13. Schrag A, Good CD, Miszkiel K, Morris HR, Mathias CJ, Lees AJ, et al. Differentiation of Atypical Parkinsonian Syndromes with Routine MRI. Neurology. 2000; 54:697-702.

14. Booij J, Speelman JD, Horstink MWIM, Wolters EC. The Clinical Benefit of Imaging Striatal Dopamine Transporter with [123I]FP-CIT SPECT in Differentiating Patients with Presynaptic Parkinsonism from those with Other Forms of Parkinsonism. Eur J Nuclear Med. 2001;28:266-72.

15. Oosterloo M, Bijlsma EK, van Kuijk SM, Minkels F, de Die-Smulders CE. Clinical and genetic characteristics of late-onset Huntington's disease. Parkinsonism Relat Disord. 2019;61:101-5. doi: 10.1016/j.parkreldis.2018.11.009.

16. Berg D, Roggendorf W, Schröder U, Klein R, Tatschner T, Benz P, et al. Echogenicity of the SN: association with increased iron content and marker for susceptibility to nigrostriatal injury. Arch Neurol. 2002;59:999-1005.

17. Postert T, Lack B, Kuhn W, Jergas M, Andrich J, Braun B, et al. Basal ganglia alterations and brain atrophy in Huntington's disease depicted by transcranial real time sonography. J Neurol Neurosurg Psychiatry. 1999;67:457-62.

18. Postert T, Eyding J, Berg D, Przuntek H, Becker G, Finger M, et al. Transcranial sonography in spinocerebellar ataxia type 3. J Neural Transm Suppl. 2004;68:123-33.

19. Walter U, Hoeppner J, Prudente-Morrissey L, Horowski S, Herpertz SC, Benecke R. Parkinson's diseaselike midbrain sonography abnormalities are frequent in depressive disorders. Brain. 2007;130:1799987.

20. Hilditch-Maguire P, Trettel F, Passani LA, Auerbach A, Persichetti F, MacDonald ME. Huntingtin: an iron-regulated protein essential for normal nuclear and perinuclear organelles. Hum Mol Genet. 2000;9:2789-97.

21. Walter U, Školoudík D. Transcranial sonography (TCS) of brain parenchyma in movement disorders: quality standards, diagnostic applications and novel technologies. Ultraschall Med. 2014;35:322-31. 
doi: $10.1055 / \mathrm{s}-0033-1356415$.

22. Rosas HD, Chen YI, Doros G, Salat DH, Chen NK, Kwong KK, et al. Alterations in brain transition metals in Huntington disease: an evolving and intricate story. Arch Neurol. 2012;69:887-93.

23. Martino D, Karnik V, Osland S, Barnes TRE, Pringsheim TM. Movement Disorders Associated With Antipsychotic Medication in People With Schizophrenia: An Overview of Cochrane Reviews and Meta-Analysis. Can J Psychiatry. 2018:706743718777392. doi: 10.1177/0706743718777392.

\section{Figures}



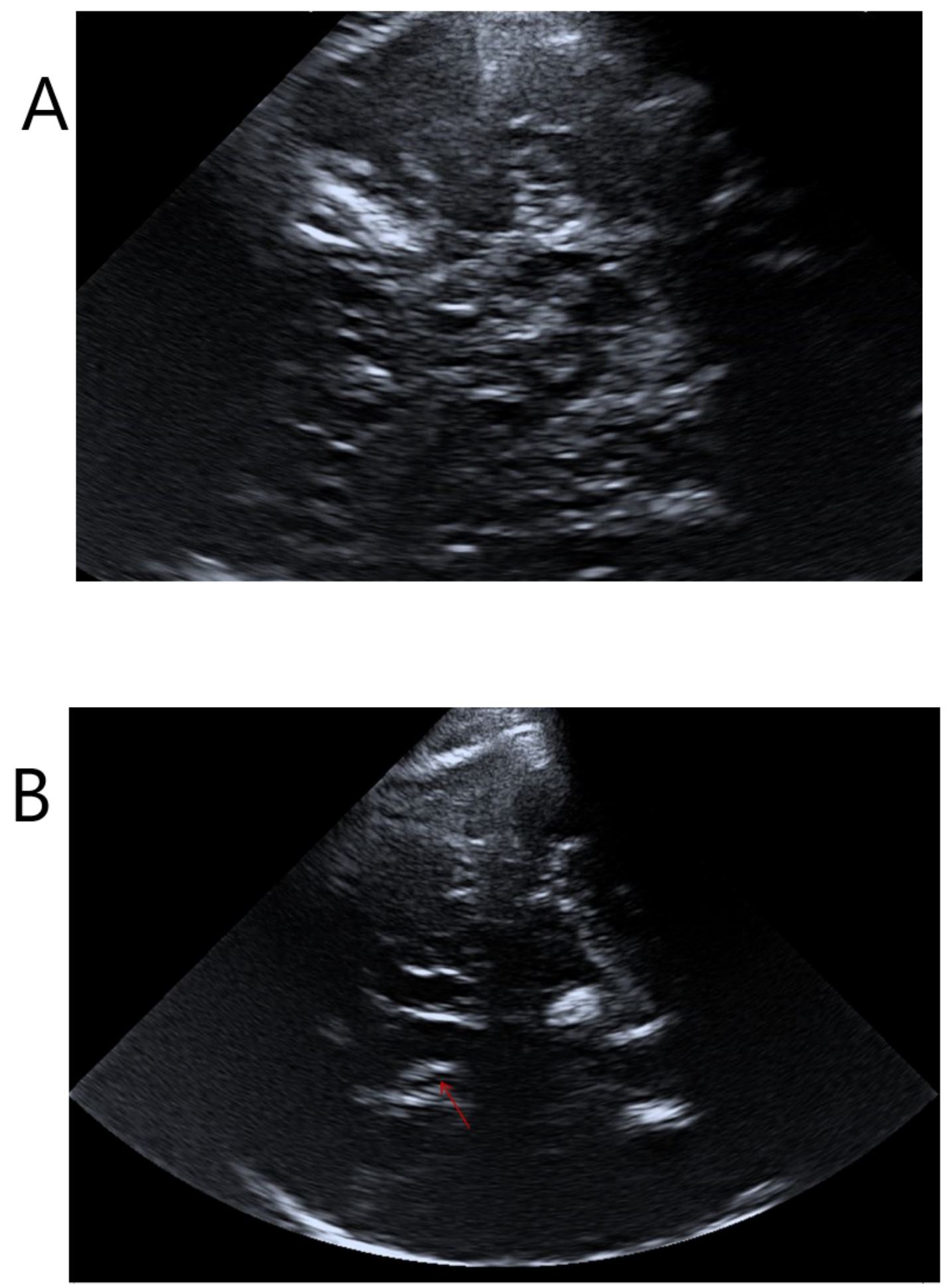

Figure 1

(A) Marked bilateral hyperechogenicity of the substantia nigra (B) Hyperechogenicity of the caudate nucleus (arrow)

\section{Supplementary Files}


This is a list of supplementary files associated with this preprint. Click to download.

- CAREchecklist.PDF 